\title{
Electrical Conductivity of Acetyl Acetonyl binylidene 5- Amino Salicylic Acid in Ethanol: Water Mixtures at Different Temperatures
}

\author{
Rana H. Al Hyali \\ Department of Chemistry / College Of Science \\ University of Mosul
}

Received
$26 / 09 / 2010$

Accepted

05 / $01 / 2011$

الخلاصة

acetyl acetonyl binyledene 5- بتضمن البحث قياس التوصيلية الكهربائية لمحلول

amino salicylic acid مختلفة (283.16º K - 313.16º

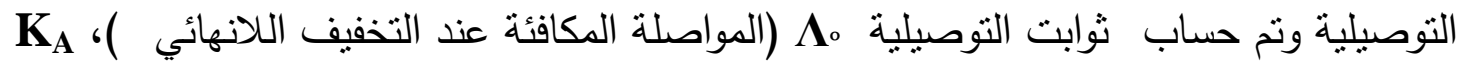

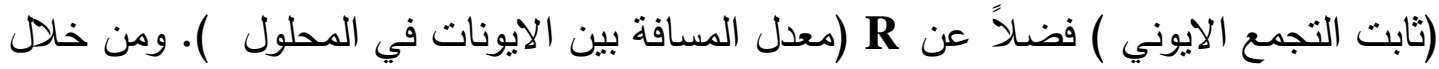

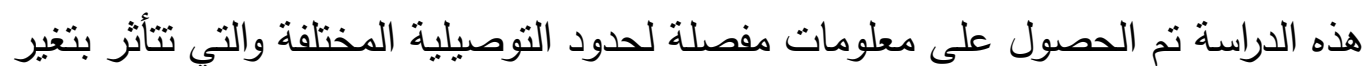

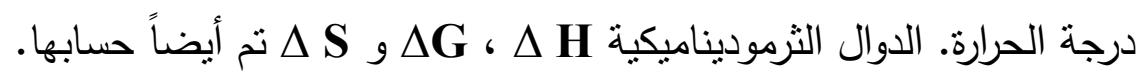

\section{ABSTRACT}

The electrical conductivity of acetyl acetonyl binyledene 5- amino salicylic acid has been measured in (ethanol: water) mixtures at different percentage and different temperatures $\left(283.16^{\circ} \mathrm{K}-313.16^{\circ} \mathrm{K}\right)$.

The results were analyzed by using Lee - Wheaton equation. The conductivity parameters, $\Lambda_{\text {}}$ ( (equivalent conductivity at infinite dilution, $\mathbf{K}_{\mathbf{A}}$ (association constant) and $\mathbf{R}$ (distance parameter between the ions) were calculated. From this study detail information about the conductivity parameters are interpreted Which have been effected by varying the temperatures. Thermodynamic parameters $\Delta \mathbf{H}, \Delta \mathbf{G}$ and $\Delta \mathbf{S}$ were also calculated.

\section{INTRODUCTION}

The measurement of electrolytic conductivities of aqueous solutions of electrolytes provides available method of studying the ionic interaction. 
Comparison of the Fuoss $1975^{(1)}$ conductance equation with previous equations shows that the only one which is based on a model which rigorously permits the use of macroscopic dielectric constant $\mathrm{D}$ and viscosity $\eta$ in the theoretical calculation of relaxation field and electophoresis.

Lee and Wheaton describe ${ }^{(2,3,4)}$ the derivation of the conductance equation based on a model identical to that suggested by Fuoss but using a new boundary condition to replace that used by Fuoss.

Conductance measurements in binary mixed solvents have been widely used for the investigation of solute - solvent interactions in electrolytes solutions. Many electrolytes solutions in different percentage of solvents mixtures and different temperatures have been studied, Ansari and Islam $^{(5)}$ studied the conductivity and the ionic association of tetraalkylammonium halides in tert-butanol-water mixture at $25^{\circ} \mathrm{C}$. Haruhiko, Tomako and Masayasu ${ }^{(6)}$ studied the conductometry of ion association between tris (ethylenediamine) cobalt(III) ion and monovalent anions at temperatures from 0 to $50{ }^{\circ} \mathrm{C}$. Tominic et.al ${ }^{(7)}$ studied the conductometry of hydrobromic acid in 2- propanol and water mixtures. Conductivity of $^{(8)}$ n-tetrabutylammonium tetraphenyl borate in 3pentanone in the temperature range from $283.15^{\circ} \mathrm{K}$ to $329.15^{\circ} \mathrm{K}$ studied by Tsierkezoz and Molinon. El-Dossoki ${ }^{(9)}$ studied the dissociation of quinic acid and association constants of some quinateions in aqueous and in alcoholic-aqueous mixed solvents.

In this work we have measured the electrical conductivity of acetyl acetonyl binyledene 5- amino salicylic acid has been measured in (ethanol: water) mixtures at different percentage and different temperatures $\left(283.16^{\circ} \mathrm{K}-313.16^{\circ} \mathrm{K}\right)$. The conductivity parameters were calculated using Lee and Wheaton equation.

\section{EXPERIMENTAL}

\section{Synthesis of acetyl acetonyl binylidine 5- amino salicylic acid:}

This compound was prepared ${ }^{10}$ By mixing 0.08 mole $(8.0 \mathrm{~g})$ of acetyl acetone with 0.16 mole $(24.48 \mathrm{~g})$ of 5 -amino salicylic acid. In $100 \mathrm{ml}$ round bottom flask, mix the quantities of acetyl acetone and the amounts of 5-amino salicylic acid mentioned. Add to the mixture $150 \mathrm{ml}$ of ethanol. The mixture was reflexed for 6 hours. The product was cooled in ice bath, following by washing the product with cold ethanol and dried.<smiles>CC(=O)CC(C)=O</smiles> 


\section{APPARATUS}

Triple distilled water was prepared by distilling water three times, ethanol was of high purity and used directly. The conductivity meter jenway PCM3 was used for measuring the resistance of the solution.

A water thermostat of type HAAKE G3 with thermo bath D3 was used for controlling the temperature of the conductance cell.

\section{Conductivity Measurments}

In clean and dry conductivity cell kept at constant temperature $298.16,100 \mathrm{ml}$ of conductivity water was placed and the conductance of the solvent was measured. Into the cell,a certain amount of the concentrated acetyl acetonyl binyledene 5- amino salicylic acid $\left(10^{-3} \mathrm{M}\right)$ in aqueous solution was injected into the cell and the conductivity of the solution was measured. This process was repeated generally for about 25 additions. The conductivity of solution was measured at different percentage of ethanol: water mixture and different temperatures by using the same process.

Tables (1-5), shows the solution molarities and the equivalent conductivities of acetyl acetonyl binyledene 5- amino salicylic acid in different percentages of $(5,10,15,20,25 \%$ ethanol: water) at different temperatures respectively. The physical properties of solvents used are listed in table (6).

Figures(1-5), illustrate the relation between the molar concentration and the equivalent conductance of acetyl acetonyl binyledene 5- amino salicylic acid in different percentages of $(5,10,15,20,25 \%$ ethanol: water) mixtures at different percentage and different temperatures, the figure (6) shows the plot of Walden product against 1/D for acetyl acetonyl binyledene 5- amino salicylic acid in (5, 10, 15, 20, 25\% ethanol: water) mixtures at $298^{\circ} \mathrm{K}$. The figures (7-11) shows the plot of the $\ln \left(\mathrm{K}_{\mathrm{A}}\right)$ against $1 / \mathrm{T}$ for acetyl acetonyl binyledene 5- amino salicylic acid solution in $(5,10,15,20,25 \%$ ethanol: water) mixtures at different temperatures.

\section{RESULTS AND DISCUSSION}

For the specific case of solutions containing only a single symmetrical electrolyte this equation has the following general form:

$$
\Lambda_{\text {equiv }}=\mathbf{f}\left(\Lambda_{\circ}, \mathbf{R}, \mathbf{K}_{\mathrm{A}}\right)
$$

where $\Lambda^{\circ}$ is equivalent conductance at infinite dilution, $R$ the distance parameter which is defined as the distance between anion and cation at which short range forces become strong enough to impede further approach of the ions allowing the formation of CIP (the formation of contact ion - pair) or SSIP (the formation of solvent - separated ion pair, $\mathrm{K}_{\mathrm{A}}$ the pairwise ion association constant. ${ }^{(11)}$ 
Lee and Wheaton ${ }^{(4)}$ consider a symmetrical electrolyte MX and they assumed that the solute to be completely dissociated into the species $\mathrm{M}^{\mathrm{n}+}$ and $\mathrm{X}^{\mathrm{n}-}$

The following general equation, Will exit between the two species:

$$
\mathrm{M}^{\mathrm{n}+}+\mathrm{X}^{\mathrm{n}-} \stackrel{\mathrm{K}_{\mathrm{A}}}{\longleftrightarrow} \mathrm{MX}
$$

and if $\mathrm{K}_{\mathrm{A}}$ is the pairwise association constant, then:

$$
\mathbf{K}_{\mathbf{A}}=[\mathbf{M X}] /\left[\mathbf{M}^{\mathbf{n}+}\right]\left[\mathbf{X}^{\mathrm{n}-}\right]
$$

The model used in this work founded on the lee and wheaton equation:

$$
\Lambda=\Lambda \cdot\left[1+C_{1}\left(\beta_{k}\right)+C 2\left(\beta_{k}\right)^{2}+C_{3}\left(\beta_{k}\right)^{3}\right]-[(\rho K / r+K R)]\left[1+C_{4}\left(\beta_{k}\right)+\right.
$$

With coefficient $\mathbf{C}_{\mathbf{1}^{-}} \mathbf{C}_{\mathbf{5}}$, as given by Pethybridge and Taba ${ }^{(12,13)}$ (model LWP), $\boldsymbol{\Lambda}$ is molar conductivity of electrolyte, $\boldsymbol{\Lambda}_{\text {。 }}$ is equivalent conductance at infinite dilution, $\beta=\mathbf{2 q}, K^{\mathbf{2}}=\mathbf{1 6 0 0 0} \pi \mathbf{N}_{\mathrm{A}} \mathbf{q} \mathbf{C} \boldsymbol{\alpha}$, where $\alpha$ is the degree of dissociation, $\mathbf{q}$ (Bjerrum parameter) $=\mathrm{e}^{2 / 8} \boldsymbol{\pi} \boldsymbol{\varepsilon}_{\circ} \boldsymbol{\varepsilon}_{\mathbf{r}} \mathbf{K T}$, where $\boldsymbol{\varepsilon}_{o}, \boldsymbol{\varepsilon}_{\mathbf{r}}$ is the permitivity, $\mathbf{R}$ is the distance parameter.

Using this expression in least - square curve fitting procedure (symmetrical) which was describe by lee and wheaton, all the terms $\Lambda_{\circ}$, $\mathbf{R}, \mathbf{K}_{\mathbf{A}}$ can be determined at the minimum value of $\boldsymbol{\sigma}(\mathbf{A})$ (standard deviation $)=\left[\Lambda\left(\Lambda_{\text {equiv }} \text { calc. }-\Lambda_{\text {equiv }}(\text { experi. })\right)^{2} \mathbf{N p}\right]^{1 / 2}$ where $\mathrm{Np}$ is the number of points of measurements.

The input data to computer program are:

Temperature (T), Dielectric constant (D), and Viscosity $\eta$, Assosiation constant $\mathrm{K}_{\mathrm{A}}$ and limiting equivalent conductivity $\Lambda_{\circ}$ and $\mathrm{R}$, together with the solution conductivities and the corresponding equivalent conductance.

In the five mixed solvent composition, the limiting equivalent conductance $\Lambda_{0}$ decrease by increasing the $\%$ wt of alcohol every the dielectric constant of the medium decreasing this is because characteristic of alcohol become more predominate ${ }^{(5)}$.

As we show in tables (1-5) the equivalent conductivity values are increase by increasing temperature this is because incrasing the kinetic energy of molecules, break up hydrogen bonds and decreasing the viscosity ${ }^{(14)}$ as we show in table(6).

Tables $(7-11)$ shows the values of $K_{A}$ incrsase with increasing the percentage of alcohol in each mixture, this due to the decreasing of the dielectric constant by increasing the percentage of alcohol ${ }^{(15)}$. In the other side $K_{A}$ values decrease with increasing temperature this because increasing the kinetic energy of molecules, break the electrostatic bonds and because the reciprocal reactions ${ }^{(16)}$. The values of $\mathrm{R}$ indicates the formation of contact ion - pair in each mixtures, The values of $\sigma(\mathrm{A})$ are 
small indicating the good applicability of the conductivity equation used L.W equation.

The case in the behaviour of the present system as indicated in figure(6) shows that the ions suffers various degree of salvation with different mixtures of ethanol and water mixtures, the value of Walden product $\left(\eta_{\circ} \Lambda_{\circ}\right)$ would be constant only if the effective radius of the ion remains the same in the different media. Hames ${ }^{(17)}$ suggest that the measure deviation in the Walden product is due to the variation of the electrochemical equilibrium between ions and the solvent molecules with the composition of the mixed polar solvents.

Thermodynamic parameters that calculated by using $\ln \mathrm{K}_{\mathrm{A}}$ and $1 / \mathrm{T}$ values that listed in table(12) shows in table (13) that $\Delta \mathrm{H}$ had negative signs, this indicated that the association reaction is exothermic, $\Delta \mathrm{G}$ values had negative signs that's referes to spontaneous the association reaction $^{(18,19)}$, while $\Delta \mathrm{S}$ values had positive signs this because of the structure of the compound in case of salvation. 
Table 1: The equivalent conductance $\Lambda\left(\Omega^{-1}\right.$. equiv $\left.{ }^{-1} . \mathrm{cm}^{2}\right)$ and the square root of molar concentration $\left(\mathrm{mol}^{\circ} \mathrm{L}^{-1}\right) * 10^{2}$ for acetyl acetonyl binyledene 5 - amino salicylic acid in a mixture of (5\% ethanol $+95 \%$ water) at $283.16{ }^{\circ} \mathrm{K}-313.16^{\circ} \mathrm{K}$

\begin{tabular}{|c|c|c|c|c|c|c|c|c|c|c|c|}
\hline \multicolumn{2}{|c|}{283.16 K } & \multicolumn{2}{|c|}{293.16 K } & \multicolumn{2}{|c|}{$298.16 \mathrm{~K}$} & \multicolumn{2}{|c|}{$303.16 \mathrm{~K}$} & \multicolumn{2}{|c|}{$308.16 \mathrm{~K}$} & \multicolumn{2}{|c|}{ 313.16 K } \\
\hline$\sqrt{\mathbf{C}}$ & $\Lambda$ & $\sqrt{C}$ & $\Lambda$ & $\sqrt{C}$ & $\Lambda$ & $\sqrt{C}$ & $\Lambda$ & $\sqrt{C}$ & $\Lambda$ & $\sqrt{C}$ & $\Lambda$ \\
\hline 0.61967 & 4.2812 & .86023 & 61.475676 & .03489 & 9.6991 & 1.03489 & 3.6501 & 0.61967 & 99.4062 & .86023 & 111.6486 \\
\hline 0.86023 & .1405 & 1.03489 & 53.5238 & 1.1743 & 1.7621 & 1.1743 & 5.1501 & 0.86023 & 1.1108 & 1.03489 & 2.7619 \\
\hline 1.03489 & 42.5714 & 1.1743 & 49.6316 & 1.29073 & 54.9795 & 1.29073 & 71.7551 & 1.03489 & 82.3809 & 1.1743 & 78.5525 \\
\hline 1.1743 & 38.4626 & 1.29073 & 46.5306 & 1.47885 & 45.9862 & 1.39104 & 66.5767 & 1.1743 & 75.2240 & 1.29073 & 63 \\
\hline 1.29073 & 35.5714 & $\mathbf{1 . 3 9 1 0 4}$ & 45.4914 & 1.55692 & 43.2995 & $\mathbf{1 . 4 7 8 8 5}$ & 60.2112 & 1.29073 & 70.7755 & .39104 & 65.6279 \\
\hline 1.39104 & 34.8961 & 1.47885 & 43.2812 & 1.62696 & 40.9618 & 1.55692 & 55.8391 & 1.39104 & 67.8418 & 1.47885 & 3.1028 \\
\hline 1.47885 & 33.9067 & 1.55692 & 41.1955 & 1.69026 & 38.8792 & 1.62696 & 52.6762 & 1.47885 & 61.7969 & 1.55692 & 62.7821 \\
\hline 1.55692 & 33.2846 & 1.62696 & 39.8058 & 1.74785 & 38.0955 & 1.69026 & 49.6254 & 1.55692 & 57.4801 & 1.62696 & 62.5024 \\
\hline 1.62696 & 4457 & $\mathbf{1 . 6 9 0 2 6}$ & 38.2366 & 1.80083 & 37.6799 & 1.74785 & 43.7715 & 1.62696 & 57.4544 & .69026 & 363 \\
\hline 1.69026 & 31.1319 & 1.74785 & 37.7617 & 1.84959 & 37.5381 & 1.80083 & 42.9639 & 1.69026 & 55.6233 & 1.74785 & 88.7968 \\
\hline 1.74785 & 30.7836 & 1.80083 & 37.1452 & 1.89446 & 36.6904 & 1.84959 & 42.0999 & 1.74785 & 54.0216 & 1.80083 & 58.3181 \\
\hline 1.80083 & 30.4144 & 1.84959 & 36.3455 & 1.93649 & 35.8561 & 1.89446 & 41.1239 & 1.84959 & 51.7603 & 1.84959 & 56.8266 \\
\hline 1.84959 & 30.6208 & 1.89446 & 35.7241 & 1.97534 & 35.3418 & 1.93649 & 40.1480 & 1.89446 & 51.4689 & .89446 & 56.2542 \\
\hline 1.89446 & 30.4664 & 1.93649 & 34 & 01171 & 35.2601 & 1.97534 & 39.7073 & 1.93649 & 51.2308 & .93649 & 55.7509 \\
\hline 1.93649 & 29.9344 & $\mathbf{1 . 9 7 5 3 4}$ & 34. & .04597 & 34.9178 & 2.01171 & 39.6204 & 1.97534 & 50.7124 & 1.97534 & 55.3616 \\
\hline 1.97534 & 29.5386 & 2.01171 & 33.8739 & 2.07797 & 34.8661 & 2.04597 & 39.4013 & 2.01171 & 50.4581 & 2.01171 & 55.3228 \\
\hline 2.01171 & 29.2868 & 2.04597 & 33.2852 & 2.1080 & 34.4284 & 2.07797 & 39.2362 & 2.04597 & 50.2689 & 2.04597 & 55.1314 \\
\hline 2.04597 & 28.8748 & 2.07797 & 33.0236 & 2.1365 & 34.2308 & 2.1080 & 39.0877 & 2.07797 & 49.8188 & 2.07797 & 54.7872 \\
\hline 2.07797 & 28.7244 & 2.1080 & 32.5693 & & & 2.1365 & 38.8113 & 2.1080 & 48.7965 & & 54.3318 \\
\hline & & 2.1365 & 32.5104 & & & 2.1633 & 38.5115 & 2.1365 & 48.6650 & & 54.1972 \\
\hline & & 2.1633 & 32.4089 & & & 2.1883 & 38.3218 & 2.1633 & 48.5153 & & 2.1080 \\
\hline & & 2.1883 & 32.3180 & & & 2.2129 & 38.1172 & 2.1883 & 48.2216 & & 2.1365 \\
\hline & & & & & & & & 2.2129 & 48.0318 & & \\
\hline
\end{tabular}


Table 2: The equivalent conductance $\Lambda\left(\Omega^{-1}\right.$. equiv $\left.{ }^{-1} . \mathrm{cm}^{2}\right)$ and the square root of molar concentration $\left(\mathrm{mol}^{-\mathrm{L}^{-1}}\right) * 10^{2}$ for acetyl acetonyl binyledene 5 - amino salicylic acid in a mixture of (10\% ethanol $+90 \%$ water) at $283.16{ }^{\circ} \mathrm{K}-313.16{ }^{\circ} \mathrm{K}$

\begin{tabular}{|c|c|c|c|c|c|c|c|c|c|c|c|}
\hline \multicolumn{2}{|c|}{283.16 K } & \multicolumn{2}{|c|}{293.16 K } & \multicolumn{2}{|c|}{$298.16 \mathrm{~K}$} & \multicolumn{2}{|c|}{ 303.16 K } & \multicolumn{2}{|c|}{$308.16 \mathrm{~K}$} & \multicolumn{2}{|c|}{$313.16 \mathrm{~K}$} \\
\hline$\sqrt{\mathbf{C}}$ & $\Lambda$ & $\sqrt{ } \mathbf{C}$ & $\Lambda$ & $\sqrt{ } \mathbf{C}$ & $\Lambda$ & $\sqrt{ } \mathbf{C}$ & $\Lambda$ & $\sqrt{C}$ & $\Lambda$ & $\sqrt{C}$ & $\Lambda$ \\
\hline 1.1743 & 39.3502 & 86023 & 4.9405 & .61967 & 65.1687 & 1.03489 & 70.5714 & 1.03489 & 73.0523 & 0.86023 & 5.1783 \\
\hline 1.29073 & 35.6326 & .03489 & 46.4761 & 0.86023 & 57.2324 & 1.1743 & 62.0580 & 1.29073 & 60.1020 & 1.03489 & 79.5238 \\
\hline 1.39104 & 32.7875 & 1.1743 & 44.0101 & 1.03489 & 55.9047 & 1.29073 & 54.0000 & 1.47885 & 49.2636 & 1.1743 & 69.6026 \\
\hline 1.47885 & 30.6419 & .29073 & 39.9183 & 1.1743 & 48.0783 & 1.39104 & 44.1736 & 1.55692 & 46.3237 & 1.29073 & 62.0204 \\
\hline 1.55692 & 28.6980 & .39104 & 37.8480 & 1.29073 & 41.8775 & 1.47885 & 40.8559 & 1.62696 & 43.3280 & 1.39104 & 50.2883 \\
\hline 1.62696 & 27.2436 & 47885 & 35.9 & $\mathbf{1 . 3 9 1 0 4}$ & 36.0573 & 1.55692 & 40.1856 & 1.69026 & 41.0 & .47885 & 6.6392 \\
\hline 1.69026 & 25.7409 & 1.55692 & 33.8316 & 1.47885 & 32.4663 & 1.62696 & 39.1507 & 1.74785 & 40.9001 & .55692 & 46.3292 \\
\hline 1.74785 & 24.9073 & 1.62696 & 33.5247 & 1.55692 & 32.2326 & 1.69026 & 36.8085 & 1.80083 & 40.7937 & 1.62696 & 46.1267 \\
\hline 1.80083 & 23.9666 & .69026 & 32.5600 & 1.62696 & 32.2145 & 1.74785 & 36.2258 & 1.84959 & 40.5793 & 1.69026 & 46.1267 \\
\hline 1.84959 & 23.3159 & .74785 & 31.6183 & 1.69026 & 31.9173 & 1.80083 & 379 & 1.89446 & 40.2998 & .74785 & 5.9083 \\
\hline 1.89446 & 23.2192 & 1.80083 & 31.0 & 1.74785 & 31.4180 & 1.84959 & 143 & 1.93649 & 39.9579 & .80083 & .7002 \\
\hline 1.93649 & 23.0620 & 1.84959 & 30.2034 & 1.80083 & $\mathbf{3 1 . 3 8 9 4}$ & $\mathbf{1 . 8 9 4 4 6}$ & 34. & $\mathbf{1 . 9 7 5 3 4}$ & 39.7857 & 1.84959 & $\mathbf{4 5 . 5 2 8 7}$ \\
\hline $\mathbf{1 . 9 7 5 3 4}$ & 22.7944 & $\mathbf{1 . 8 9 4 4 6}$ & 30.1538 & 1.84959 & $\mathbf{3 1 . 1 2 7 7}$ & 1.93649 & 34.3078 & 2.01171 & 39.2928 & 1.89446 & 45.3017 \\
\hline 2.01171 & 22.7086 & 1.93649 & 29.7443 & 1.89446 & 30.8643 & $\mathbf{1 . 9 7 5 3 4}$ & 34.1655 & 2.04597 & 39.2064 & 1.93649 & 45.2005 \\
\hline 2.04597 & 22.2957 & $\mathbf{1 . 9 7 5 3 4}$ & 29.6955 & 1.93649 & 30.5592 & 2.01171 & 33.9747 & 2.07797 & 39.0236 & 1.97534 & 5.0661 \\
\hline 2.07797 & 22.2283 & 2.01171 & 29.3624 & 1.97534 & 30.3751 & 2.04597 & 33.6751 & 2.10807 & 38.9500 & 2.01171 & 44.9636 \\
\hline 2.1080 & 22.1489 & 2.04597 & 28.8260 & 2.01171 & 30.3202 & 2.07797 & $\mathbf{3 3 . 3 3 0 7}$ & 2.1365 & 38.8560 & 2.04597 & 44.6889 \\
\hline 2.1365 & 22.0534 & 2.07797 & 28.6299 & 2.04597 & 30.1419 & 2.10807 & 33.1201 & 2.1633 & 38.7294 & 2.07797 & 44.5039 \\
\hline & & 2.1080 & 28.4608 & 2.07797 & 29.9527 & 2.1633 & 32.8666 & 2.1883 & 38.3218 & 2.10807 & 4.3897 \\
\hline & & 2.1365 & 28.2203 & 2.10807 & 29.6314 & 2.1883 & 32.5948 & 2.21291 & 38.3046 & 2.13658 & 4.1069 \\
\hline & & 2.1633 & 28.2243 & & & 2.2129 & 32.4725 & 2.23606 & 38.1276 & 2.16333 & 43.8948 \\
\hline & & & & & & 2.23606 & 32.3952 & & & 2.1883 & 43.7294 \\
\hline & & & & & & & & & & 2.21291 & 43.6160 \\
\hline & & & & & & & & & & 2.23606 & 43.5336 \\
\hline
\end{tabular}


Table 3: The equivalent conductance $\Lambda\left(\Omega^{-1}\right.$. equiv $\left.{ }^{-1} . \mathrm{cm}^{2}\right)$ and the square root of molar concentration $\left(\mathrm{mol}^{1} \mathrm{~L}^{-1}\right) * 10^{2}$ for acetyl acetonyl binyledene 5 - amino salicylic acid in a mixture of (15\% ethanol $+85 \%$ water) at $283.16^{\circ} \mathrm{K}-313.16^{\circ} \mathrm{K}$

\begin{tabular}{|c|c|c|c|c|c|c|c|c|c|c|c|}
\hline \multicolumn{2}{|c|}{283.16 K } & \multicolumn{2}{|c|}{293.16 K } & \multicolumn{2}{|c|}{298.16 K } & \multicolumn{2}{|c|}{$303.16 \mathrm{~K}$} & \multicolumn{2}{|c|}{$308.16 \mathrm{~K}$} & \multicolumn{2}{|c|}{$313.16 \mathrm{~K}$} \\
\hline$\sqrt{C}$ & $\Lambda$ & $\sqrt{C}$ & $\Lambda$ & $\sqrt{C}$ & $\Lambda$ & $\sqrt{C}$ & $\Lambda$ & $\sqrt{\mathbf{C}}$ & $\Lambda$ & $\sqrt{C}$ & $\Lambda$ \\
\hline 0.61967 & 45.6368 & 0.61967 & 49.2187 & 0.86023 & $\mathbf{5 8 . 8 8 3 7}$ & 0.86023 & 58.0567 & 1.03489 & 67.14285 & 0.86023 & 93.984 \\
\hline 0.86023 & 38.5945 & 0.86023 & 45.2108 & 1.03489 & 52.3809 & 1.1743 & 52.8861 & 1.1743 & 59.83901 & 1.03489 & 74.4761 \\
\hline 1.03489 & 33.0476 & 1.03489 & 37.7142 & 1.1743 & 42.4568 & 1.29073 & 48.7959 & 1.29073 & 50.08163 & 1.1743 & 62.9456 \\
\hline 1.1743 & 28.4771 & 1.1743 & 34.0986 & 1.29073 & 36.4285 & 1.39104 & 43.1720 & 1.39104 & 44.75348 & 1.29073 & 54.4285 \\
\hline 1.29073 & 26.6938 & 1.29073 & 29.3265 & 1.39104 & 33.6837 & 1.47885 & 39.0370 & 1.47885 & 41.78875 & 1.39104 & 47.8635 \\
\hline 1.39104 & 24.0372 & $\mathbf{1 . 3 9 1 0 4}$ & 26.4620 & 1.47885 & 31.0617 & $\mathbf{1 . 5 5 6 9 2}$ & 36.3985 & $\mathbf{1 . 5 5 6 9 2}$ & 38.67079 & 1.47885 & 42.6748 \\
\hline 1.47885 & 23.8792 & 1.47885 & 24.3923 & 1.55692 & 29.1608 & 1.62696 & 33.7944 & 1.62696 & 35.72119 & 1.55692 & 39.5965 \\
\hline 1.55692 & 22.5123 & 1.55692 & 24.2376 & 1.62696 & 27.5519 & 1.69026 & 31.8102 & 1.69026 & 33.95239 & 1.62696 & 36.8386 \\
\hline 1.62696 & 21.3479 & 1.62696 & 24.0838 & 1.69026 & 26.2765 & 1.74785 & 30.8504 & 1.74785 & 33.62160 & 1.69026 & 36.7371 \\
\hline 1.69026 & 21.2068 & 1.69026 & 24.0273 & 1.74785 & 25.8422 & 1.80083 & 29.9740 & 1.80083 & 33.40240 & 1.74785 & 36.4929 \\
\hline 1.74785 & 21.0677 & 1.74785 & 23.5384 & 1.80083 & 25.6336 & 1.84959 & 29.7562 & 1.84959 & 33.00613 & 1.80083 & 36.2960 \\
\hline 1.80083 & 20.3811 & 1.80083 & 22.8344 & 1.84959 & 25.2838 & 1.89446 & 29.5001 & 1.89446 & 32.93898 & 1.84959 & 36.0473 \\
\hline 1.84959 & 20.1555 & 1.84959 & 22.4215 & 1.93649 & 25.1234 & 1.93649 & 29.3640 & 1.93649 & 32.51504 & 1.89446 & 35.8662 \\
\hline 1.89446 & 20.1214 & 1.89446 & 22.1677 & 1.97534 & 24.8820 & 1.97534 & 29.1727 & 1.97534 & 32.12660 & 1.93649 & 35.5573 \\
\hline 1.93649 & 19.9925 & 1.93649 & 21.8940 & 2.01171 & 24.8595 & 2.01171 & 29.0096 & 2.01171 & 32.00889 & $\mathbf{1 . 9 7 5 3 4}$ & 35.3418 \\
\hline 1.97534 & 19.8928 & 1.97534 & 21.5397 & 2.04597 & 24.3469 & 2.04597 & 28.8017 & 2.04597 & 31.99378 & 2.01171 & 35.1593 \\
\hline 2.01171 & 19.5833 & 2.01171 & 21.1712 & & 24.1963 & 2.07797 & 28.5590 & 2.07797 & 31.62992 & 2.04597 & 34.8690 \\
\hline 2.04597 & 19.5422 & 2.04597 & 21.0774 & & & 2.10807 & 28.3001 & 2.10807 & 31.19216 & 2.07797 & 34.6535 \\
\hline 2.07797 & 19.2519 & & & & & 2.13658 & 28.1756 & 2.13658 & 30.49945 & 2.10807 & 34.3366 \\
\hline 2.10807 & 18.7979 & & & & & 2.16333 & 28.0717 & 2.16333 & 30.33846 & 2.13658 & 34.2308 \\
\hline 2.13658 & 18.6348 & & & & & 2.1883 & 28.0388 & 2.1883 & 30.14652 & 2.16333 & 34.0435 \\
\hline 2.16333 & 18.3512 & & & & & 2.21291 & 27.9317 & 2.21291 & 30.01470 & 2.1883 & 33.8509 \\
\hline & & & & & & 2.23606 & 27.8868 & & & 2.21291 & 33.6389 \\
\hline & & & & & & & & & & 2.23606 & 33.4764 \\
\hline
\end{tabular}


Table 4: The equivalent conductance $\Lambda\left(\Omega^{-1}\right.$. equiv $\left.{ }^{-1} . \mathrm{cm}^{2}\right)$ and the square root of molar concentration $\left(\mathrm{mol}^{-1} \mathrm{~L}^{-1}\right) * 10^{2}$ for acetyl acetonyl binyledene 5 - amino salicylic acid in a mixture of $(20 \%$ ethanol $+80 \%$ water $)$ at $283.16{ }^{\circ} \mathrm{K}-313.16{ }^{\circ} \mathrm{K}$

\begin{tabular}{|c|c|c|c|c|c|c|c|c|c|c|c|}
\hline \multicolumn{2}{|c|}{$283.16 \mathrm{~K}$} & \multicolumn{2}{|c|}{$293 \mathrm{~K}$} & \multicolumn{2}{|c|}{$298 \mathrm{~K}$} & \multicolumn{2}{|c|}{$303 \mathrm{~K}$} & \multicolumn{2}{|c|}{$308 \mathrm{~K}$} & \multicolumn{2}{|c|}{$313 \mathrm{~K}$} \\
\hline$\sqrt{\mathbf{C}}$ & $\Lambda$ & $\sqrt{ } \mathbf{C}$ & $\Lambda$ & $\sqrt{ } \mathbf{C}$ & $\Lambda$ & $\sqrt{C}$ & $\Lambda$ & $\sqrt{ } \mathbf{C}$ & $\Lambda$ & $\sqrt{\mathbf{C}}$ & $\Lambda$ \\
\hline 0.61967 & 44.0156 & 0.61967 & 45.5125 & 0.86023 & 51.5100 & 0.86023 & 52.1459 & 0.86023 & 52.1459 & 0.86023 & 52.1459 \\
\hline 0.86023 & $\mathbf{3 1 . 4 2 7}$ & 0.86023 & 38.3513 & 1.03489 & 46.9380 & 1.1743 & 44.4174 & 1.1743 & 44.4174 & 1.1743 & 44.4174 \\
\hline 1.03489 & 22.4761 & 1.03489 & 30.9523 & 1.39104 & 30.6790 & 1.29073 & 40.3061 & 1.29073 & 40.3061 & 1.29073 & 40.3061 \\
\hline 1.1743 & 17.5982 & 1.1743 & 25.3705 & 1.47885 & 28.7297 & 1.39104 & 37.0031 & 1.39104 & 37.0031 & 1.39104 & 37.0031 \\
\hline 1.29073 & 17.7142 & 1.29073 & 23.3877 & 1.55692 & 27.5618 & 1.47885 & 34.9327 & 1.47885 & 34.9327 & 1.47885 & 34.9327 \\
\hline $\mathbf{1 . 3 9 1 0 4}$ & 17.9767 & 1.39104 & 22.5085 & 1.62696 & 25.9335 & 1.55692 & 32.1064 & 1.55692 & 32.1064 & 1.55692 & 32.1064 \\
\hline 1.47885 & 16.3497 & 1.55692 & 20.6608 & 1.69026 & 24.8484 & 1.62696 & 30.0566 & 1.62696 & 30.0566 & 1.62696 & 30.0566 \\
\hline 1.55692 & 16.2873 & 1.62696 & 20.2304 & 1.74785 & 23.7387 & 1.69026 & 28.1687 & 1.69026 & 28.1687 & 1.69026 & 28.1687 \\
\hline 1.62696 & 16.2228 & 1.69026 & 20.1715 & 1.80083 & 22.9287 & 1.74785 & 26.6435 & 1.74785 & 26.6435 & 1.74785 & 26.6435 \\
\hline 1.69026 & 15.7801 & $\mathbf{1 . 7 4 7 8 5}$ & $\mathbf{1 9 . 8 3 2 4}$ & 1.84959 & 22.2128 & 1.80083 & 25.3506 & 1.80083 & 25.3506 & 1.80083 & 25.3506 \\
\hline 1.74785 & 15.6255 & 1.80083 & 19.5948 & 1.89446 & 21.5140 & 1.84959 & 24.9260 & 1.84959 & 24.9260 & 1.84959 & 24.9260 \\
\hline 1.80083 & 15.1285 & 1.84959 & 19.3504 & 1.93649 & 20.9976 & 1.89446 & 24.7255 & 1.89446 & 24.7255 & 1.89446 & 24.7255 \\
\hline 1.84959 & 14.9079 & 1.89446 & 19.3257 & 1.97534 & 20.8077 & 1.93649 & 24.4202 & 1.93649 & 24.4202 & 1.93649 & 24.4202 \\
\hline 1.89446 & 14.6079 & 1.93649 & 19.0418 & 2.01171 & 20.6167 & 1.97534 & 24.1537 & $\mathbf{1 . 9 7 5 3 4}$ & 24.1537 & 1.97534 & 24.1537 \\
\hline 1.93649 & 14.3424 & 1.97534 & 18.9518 & 2.04597 & 20.5900 & 2.01171 & 23.9688 & 2.01171 & 23.9688 & 2.01171 & 23.9688 \\
\hline 1.97534 & 14.2988 & 2.01171 & 18.6256 & 2.10807 & 20.1291 & 2.04597 & 23.8552 & 2.04597 & 23.8552 & 2.04597 & 23.8552 \\
\hline 2.01171 & 14.1645 & 2.04597 & 18.1046 & 2.13658 & 19.9978 & 2.07797 & 23.7401 & 2.07797 & 23.7401 & 2.07797 & 23.7401 \\
\hline 2.04597 & 14.1328 & 2.04597 & 18.1046 & 2.16333 & $\mathbf{1 9 . 8 1 1 5}$ & 2.10807 & 23.6638 & 2.10807 & 23.6638 & 2.10807 & 23.6638 \\
\hline 2.10807 & 13.7943 & & & 2.1883 & 19.6080 & 2.13658 & 23.4387 & 2.13658 & 23.4387 & 2.13658 & 23.4387 \\
\hline 2.13658 & 13.6521 & & & 2.21291 & 19.5793 & 2.16333 & 23.3858 & 2.16333 & 23.3858 & 2.16333 & 23.3858 \\
\hline 2.16333 & 13.4692 & & & 2.23606 & $\mathbf{1 9 . 3 5 9 6}$ & & & & & & \\
\hline 2.1883 & 13.3061 & & & 2.21291 & $\mathbf{1 9 . 5 7 9 3}$ & & & & & & \\
\hline & & & & 2.23606 & 19.3596 & & & & & & \\
\hline
\end{tabular}


Table 5: The equivalent conductance $\Lambda\left(\Omega^{-1}\right.$. equiv $\left.{ }^{-1} . \mathrm{cm}^{2}\right)$ and the square root of molar concentration $\left(\mathrm{mol}^{-\mathrm{L}^{-1}}\right) * 10^{2}$ for acetyl acetonyl binyledene 5 - amino salicylic acid in a mixture of $(25 \%$ ethanol $+75 \%$ water $)$ at $283.16{ }^{\circ} \mathrm{K}-313.16{ }^{\circ} \mathrm{K}$

\begin{tabular}{|c|c|c|c|c|c|c|c|c|c|c|c|}
\hline \multicolumn{2}{|c|}{$283.16 \mathrm{~K}$} & \multicolumn{2}{|c|}{293.16 K } & \multicolumn{2}{|c|}{$298.16 \mathrm{~K}$} & \multicolumn{2}{|c|}{ 303.16 K } & \multicolumn{2}{|c|}{$308.16 \mathrm{~K}$} & \multicolumn{2}{|c|}{$313.16 \mathrm{~K}$} \\
\hline$\sqrt{\mathbf{C}}$ & $\Lambda$ & $\sqrt{C}$ & $\Lambda$ & $\sqrt{\mathbf{C}}$ & $\Lambda$ & $\sqrt{\mathbf{C}}$ & $\Lambda$ & $\sqrt{C}$ & $\Lambda$ & $\sqrt{C}$ & $\Lambda$ \\
\hline 1.03489 & 0.4761 & .6197 & .3221 & 86023 & 44.7142 & 86023 & 44.7142 & 86023 & 55.6837 & 0.8602 & 0.1729 \\
\hline 1.1743 & $\mathbf{1 7 . 5 3 0 0}$ & 86023 & & 1743 & 38.0159 & 1743 & 38.0159 & 1.03489 & & 1.1743 & \\
\hline 1.29073 & 15.3673 & 03489 & 27.1904 & 1.29073 & 32.5918 & 1.29073 & $\mathbf{3 2 . 5 9 1 8}$ & 1.1743 & 41.8651 & 1.29073 & 38.3265 \\
\hline 1.39104 & 13.8635 & 1.1743 & 23.1515 & 1.39104 & 29.9937 & 1.39104 & 29.9937 & 1.29073 & & 1.39104 & 33.7364 \\
\hline 1.47885 & 13.0589 & 29073 & & 1.47885 & 26.7709 & 1.47885 & 26.7709 & 39104 & & .47885 & \\
\hline 1.55692 & & 104 & & 5692 & 21 & 55692 & 24 & 47885 & & 62696 & \\
\hline 1.62696 & 12.0 & 17885 & & 1.62696 & 22.8507 & 1.62696 & 22.8507 & .55692 & & .69026 & 482 \\
\hline 1.69026 & & 55692 & & 1.69026 & & 9026 & 21.5 & .62696 & & 1.74785 & \\
\hline 1.74785 & 11.2 & 2696 & & 1.74785 & 21.2 & 4785 & 21.2 & 9026 & & 84959 & 356 \\
\hline 1.80083 & & 026 & & 80083 & & 0083 & 20. & 4785 & & 89446 & \\
\hline 1.84959 & 31 & 785 & & 1.84959 & 20.3940 & .84959 & 20.3940 & .80083 & 591 & 93649 & 375 \\
\hline 1.89446 & & 1.80083 & & 1.89446 & 20.1499 & 89446 & 20.1499 & .84959 & 265 & 1.97534 & 24.5197 \\
\hline 1.93649 & $\mathbf{1 0 . 7 0 2 5}$ & .84959 & 16.3688 & 1.93649 & 20.0468 & 93649 & 20.0468 & .89446 & 1908 & 2.01171 & 24.3217 \\
\hline $\mathbf{1 . 9 7 5 3 4}$ & $\mathbf{1 0 . 5 6 0 7}$ & 89446 & 142 & $\mathbf{1 . 9 7 5 3 4}$ & 9.8928 & 97534 & $\mathbf{1 9 . 8 9 2 8}$ & 93649 & 348 & 2.04597 & 24.2694 \\
\hline 2.01171 & 10.2579 & 3649 & & 2.01171 & 19.8606 & 01171 & 19.8606 & 97534 & & 07797 & 4.0472 \\
\hline 2.04597 & & 1.97534 & & 2.04597 & 19.7372 & 4597 & 19.7372 & 1171 & 078 & 2.10807 & 24.0310 \\
\hline 2.07797 & & 117 & 15.5003 & 2.07797 & 19.5826 & 2.07797 & 19.5826 & 4597 & 932 & 2.13658 & 23.8633 \\
\hline 2.1080 & & 597 & 87 & 2.10807 & & 2.10807 & & 7797 & & 2.16333 & \\
\hline 2.1365 & & & & 2.13658 & 19.3721 & 2.13658 & & 0807 & & 2.1883 & \\
\hline 2.1633 & 9.5243 & & & 2.16333 & 19.2230 & 2.16333 & 19.2230 & 2.13658 & 22.0087 & 2.21291 & 23.1202 \\
\hline & & & & & & & & 2.16333 & 21.8166 & & \\
\hline & & & & & & & & 2.1883 & 21.6731 & & \\
\hline
\end{tabular}


Table 6: Viscosity and Dielectric constant of ethanol: water mixture at different .temperatures

\begin{tabular}{|c|c|c|c|c|c|c|}
\hline \multicolumn{7}{|c|}{$5 \%$ ethanol + 95\% water } \\
\hline & $283^{\circ} \mathrm{K}$ & $293^{\circ} \mathrm{K}$ & $298^{\circ} \mathrm{K}$ & $303^{\circ} \mathrm{K}$ & $308^{\circ} \mathrm{K}$ & $313^{\circ} \mathrm{K}$ \\
\hline $\begin{array}{l}\text { Viscosity } \\
\text { Kg.m }^{-1} \mathrm{~s}^{-1}\end{array}$ & 0.01314 & 0.010119 & 0.008978 & 0.008077 & 0.007289 & 0.006619 \\
\hline $\begin{array}{l}\text { Dielectric } \\
\text { constant }\end{array}$ & 80.97 & 77.4 & 75.6 & 73.9 & 72.3 & 70.6 \\
\hline \multicolumn{7}{|c|}{$10 \%$ ethanol + $90 \%$ water } \\
\hline & $283^{\circ} \mathrm{K}$ & $293^{\circ} \mathrm{K}$ & $298^{\circ} \mathrm{K}$ & $303^{\circ} \mathrm{K}$ & $308^{\circ} \mathrm{K}$ & $313^{\circ} \mathrm{K}$ \\
\hline $\begin{array}{l}\text { Viscosity } \\
\mathrm{Kg} \cdot \mathrm{m}^{-1} \mathrm{~s}^{-1}\end{array}$ & 0.01322 & 0.010218 & 0.009053 & 0.008180 & 0.007385 & 0.006670 \\
\hline $\begin{array}{l}\text { Dielectric } \\
\text { constant }\end{array}$ & 78.1 & 74.7 & 72.9 & 71.3 & 69.7 & 68.0 \\
\hline \multicolumn{7}{|c|}{$15 \%$ ethanol $+85 \%$ water } \\
\hline & $283^{\circ} \mathrm{K}$ & $293^{\circ} \mathrm{K}$ & $298^{\circ} \mathrm{K}$ & $303^{\circ} \mathrm{K}$ & $308^{\circ} \mathrm{K}$ & $313^{\circ} \mathrm{K}$ \\
\hline $\begin{array}{l}\text { Viscosity } \\
\mathrm{Kg} \cdot \mathrm{m}^{-1} \mathrm{~s}^{-1}\end{array}$ & 0.01330 & 0.010317 & 0.009128 & 0.008283 & 0.007481 & 0.006740 \\
\hline $\begin{array}{l}\text { Dielectric } \\
\text { constant }\end{array}$ & 75.3 & 71.9 & 70.2 & 68.6 & 67.1 & 65.443 \\
\hline \multicolumn{7}{|c|}{$20 \%$ ethanol $+80 \%$ water } \\
\hline & $283^{\circ} \mathrm{K}$ & $293^{\circ} \mathrm{K}$ & $298^{\circ} \mathrm{K}$ & $303^{\circ} \mathrm{K}$ & $308^{\circ} \mathrm{K}$ & $313^{\circ} \mathrm{K}$ \\
\hline $\begin{array}{l}\text { Viscosity } \\
\mathrm{Kg} \cdot \mathrm{m}^{-1} \mathrm{~s}^{-1}\end{array}$ & 0.01338 & 0.010416 & 0.009203 & 0.008386 & 0.007577 & 0.006811 \\
\hline $\begin{array}{l}\text { Dielectric } \\
\text { constant }\end{array}$ & 72.4 & 69.2 & 67.5 & 66.0 & 64.6 & 62.9 \\
\hline \multicolumn{7}{|c|}{$25 \%$ ethanol $+75 \%$ water } \\
\hline & $283^{\circ} \mathrm{K}$ & $293^{\circ} \mathrm{K}$ & $298^{\circ} \mathrm{K}$ & $303^{\circ} \mathrm{K}$ & $308^{\circ} \mathrm{K}$ & $313^{\circ} \mathrm{K}$ \\
\hline $\begin{array}{l}\text { Viscosity } \\
\text { Kg. } \mathrm{m}^{-1} \mathrm{~s}^{-1}\end{array}$ & 0.01346 & 0.010515 & 0.009278 & 0.008488 & 0.007673 & 0.006888 \\
\hline $\begin{array}{l}\text { Dielectric } \\
\text { constant }\end{array}$ & 69.5 & 66.5 & 64.8 & 63.4 & 62.0 & 60.3 \\
\hline
\end{tabular}

Table 7: $\Lambda_{\circ}, K_{\mathrm{A}}, \mathbf{R}$ and $\sigma$ (A) for acetonyl binyledene 5- amino salicylic acid in $\left(5 \%\right.$ ethanol $+95 \%$ water) mixture at $283.16^{\circ} \mathrm{K}-313.16^{\circ} \mathrm{K}$.

\begin{tabular}{|c|c|c|c|c|}
\hline $\mathbf{T}(\mathbf{K})$ & $\boldsymbol{\Lambda}_{\circ}$ & $\mathbf{K}_{\mathbf{A}}$ & $\mathbf{R}\left(\mathbf{A}^{\mathbf{0}^{\mathbf{0}}}\right.$ & $\boldsymbol{\sigma}(\mathbf{A})$ \\
\hline 283.16 & 80.334 & 13503.526 & 2 & 0.017 \\
\hline 293.16 & 97.902 & 13469.909 & 2 & 0.036 \\
\hline 298.16 & 101.221 & 12792.639 & 2.1 & 0.047 \\
\hline 303.16 & 113.036 & 12314.768 & 2.1 & 0.064 \\
\hline 308.16 & 137.476 & 11829.684 & 2 & 0.027 \\
\hline 313.16 & 150.291 & 11568.877 & 2 & 0.033 \\
\hline
\end{tabular}


Table 8: $\Lambda_{\circ}, K_{\mathrm{A}}, R$ and $\sigma(A)$ for acetonyl binyledene 5- amino salicylic acid in $\left(10 \%\right.$ ethanol $+90 \%$ water) mixture at $283.16^{\circ} \mathrm{K}-313.16^{\circ} \mathrm{K}$.

\begin{tabular}{|c|c|c|c|c|}
\hline $\mathbf{T}(\mathbf{K})$ & $\boldsymbol{\Lambda}_{\mathbf{0}}$ & $\mathbf{K}_{\mathbf{A}}$ & $\mathbf{R}\left(\mathbf{A}^{\circ}\right)$ & $\boldsymbol{\sigma}(\mathbf{A})$ \\
\hline 283.16 & 78.291 & 32687.297 & 2.1 & 0.042 \\
\hline 293.16 & 93.291 & 17997.181 & 2 & 0.066 \\
\hline 298.16 & 94.877 & 17392.300 & 2 & 0.023 \\
\hline 303.16 & 107.103 & 15275.268 & 2 & 0.035 \\
\hline 308.16 & 120.294 & 15143.531 & 2 & 0.024 \\
\hline 313.16 & 123.238 & 11905.800 & 2 & 0.046 \\
\hline
\end{tabular}

Table 9: $\Lambda_{\circ}, K_{\mathrm{A}}, \mathbf{R}$ and $\sigma$ (A) for acetonyl binyledene 5- amino salicylic acid in $\left(15 \%\right.$ ethanol $+85 \%$ water) mixture at $283.16^{\circ} \mathrm{K}-313.16^{\circ} \mathrm{K}$.

\begin{tabular}{|c|c|c|c|c|}
\hline $\mathbf{T}(\mathbf{K})$ & $\boldsymbol{\Lambda}_{\mathbf{o}}$ & $\mathbf{K}_{\mathbf{A}}$ & $\mathbf{R}\left(\mathbf{A}^{\mathbf{0}}\right)$ & $\boldsymbol{\sigma}(\mathbf{A})$ \\
\hline 283.16 & 76.495 & 30347.975 & 2 & 0.071 \\
\hline 293.16 & 84.267 & 29797.746 & 2 & 0.013 \\
\hline 298.16 & 85.979 & 25028.226 & 2 & 0.053 \\
\hline 303.16 & 105.993 & 21959.535 & 2 & 0.021 \\
\hline 308.16 & 115.361 & 21364.948 & 2.1 & 0.030 \\
\hline 313.16 & 117.526 & 16500.401 & 2.2 & 0.059 \\
\hline
\end{tabular}

Table 10: $\Lambda_{\circ}, K_{\mathrm{A}}, \mathrm{R}$ and $\sigma(\mathrm{A})$ for acetonyl binyledene 5 - amino salicylic acid in $\left(20 \%\right.$ ethanol $+80 \%$ water)mixture at $283.16^{\circ} \mathrm{K}-313.16^{\circ} \mathrm{K}$.

\begin{tabular}{|c|c|c|c|c|}
\hline $\mathbf{T}(\mathbf{K})$ & $\boldsymbol{\Lambda}_{\mathbf{o}}$ & $\mathbf{K}_{\mathbf{A}}$ & $\mathbf{R}\left(\mathbf{A}^{\mathbf{0}}\right)$ & $\boldsymbol{\sigma}(\mathbf{A})$ \\
\hline 283.16 & 70.398 & 48892.998 & 2 & 0.019 \\
\hline 293.16 & 82.560 & 42277.029 & 2 & 0.011 \\
\hline 298.16 & 85.553 & 38706.509 & 2.1 & 0.048 \\
\hline 303.16 & 109.094 & 34849.735 & 2 & 0.018 \\
\hline 308.16 & 111.154 & 30201.613 & 2.1 & 0.032 \\
\hline 313.16 & 115.150 & 26610.890 & 2.1 & 0.037 \\
\hline
\end{tabular}

Table 11: $\Lambda \circ, K_{\mathrm{A}}, \mathbf{R}$ and $\sigma(\mathrm{A})$ for acetonyl binyledene 5 - amino salicylic acid in $\left(25 \%\right.$ ethanol $+75 \%$ water) mixture at $283.16^{\circ} \mathrm{K}-313.16^{\circ} \mathrm{K}$.

\begin{tabular}{|c|c|c|c|c|}
\hline $\mathbf{T}(\mathbf{K})$ & $\boldsymbol{\Lambda}_{\mathbf{*}}$ & $\mathbf{K}_{\mathbf{A}}$ & $\mathbf{R}\left(\mathbf{A}^{\mathbf{0}}\right)$ & $\boldsymbol{\sigma}(\mathbf{A})$ \\
\hline 283.16 & 61.898 & 74073.408 & 2 & 0.051 \\
\hline 293.16 & 80.145 & 55918.429 & 2 & 0.017 \\
\hline 298.16 & 84.247 & 40994.235 & 2 & 0.017 \\
\hline 303.16 & 93.636 & 38851.117 & 2 & 0.016 \\
\hline 308.16 & 101.694 & 32932.044 & 2.69 & 0.028 \\
\hline 313.16 & 103.858 & 31395.991 & 2 & 0.027 \\
\hline
\end{tabular}


Table 12: $\ln \mathrm{K}_{\mathrm{A}}$ and $(1 / \mathrm{T})^{*} 10^{3} \mathrm{~K}^{-1}$ for acetonyl binyledene 5- amino salicylic acid in ethanol: water) mixtures at different temperatures.

\begin{tabular}{|c|c|c|c|c|c|c|}
\hline \multicolumn{7}{|c|}{$5 \%$ ethanol $+95 \%$ water } \\
\hline $\ln K_{A}$ & 9.510 & 9.508 & 9.456 & 9.418 & 9.378 & 9.356 \\
\hline$(1 / T)^{*} 10^{3} K^{-1}$ & 3.531 & 3.411 & 3.353 & 3.298 & 3.245 & 3.193 \\
\hline \multicolumn{7}{|c|}{$10 \%$ ethanol $+90 \%$ water } \\
\hline $\ln K_{A}$ & 10.394 & 9.797 & 9.763 & 9.633 & 9.625 & 9.384 \\
\hline$(1 / \mathrm{T})^{*} 10^{3} \mathrm{~K}^{-1}$ & 3.531 & 3.411 & 3.353 & 3.298 & 3.245 & 3.193 \\
\hline \multicolumn{7}{|c|}{$15 \%$ ethanol $+85 \%$ water } \\
\hline $\ln K_{A}$ & 10.320 & 10.302 & 10.127 & 9.996 & 9.969 & 9.711 \\
\hline$(1 / \mathrm{T})^{*} 10^{3} \mathrm{~K}^{-1}$ & 3.531 & 3.411 & 3.353 & 3.298 & 3.245 & 3.193 \\
\hline \multicolumn{7}{|c|}{$20 \%$ ethanol $+80 \%$ water } \\
\hline $\ln K_{A}$ & 10.797 & 10.651 & 10.563 & 10.458 & 10.315 & 10.189 \\
\hline$(1 / T)^{*} 10^{3} K^{-1}$ & 3.531 & 3.411 & 3.353 & 3.298 & 3.245 & 3.193 \\
\hline \multicolumn{7}{|c|}{$25 \%$ ethanol $+75 \%$ water } \\
\hline $\ln K_{A}$ & 11.212 & 10.931 & 10.621 & 10.567 & 10.402 & 10.354 \\
\hline$(1 / T)^{*} 10^{3} K^{-1}$ & 3.531 & 3.411 & 3.353 & 3.298 & 3.245 & 3.193 \\
\hline
\end{tabular}

Table 13: Thermodynamic parameters of acetyl acetonyl binyledene 5- amino salicylic acid solution in ethanol: water mixtures and different temperatures.

\begin{tabular}{|c|c|c|c|c|c|c|}
\hline \multicolumn{7}{|c|}{$5 \%$ ethanol + 95\% water } \\
\hline & $283^{\circ} \mathrm{K}$ & $293^{\circ} \mathrm{K}$ & $298^{\circ} \mathrm{K}$ & $303^{\circ} \mathrm{K}$ & $308^{\circ} \mathrm{K}$ & $313^{\circ} \mathrm{K}$ \\
\hline$-\Delta \mathrm{H}\left(\mathrm{KJ} \cdot \mathrm{mol}^{-1}\right)$ & 4.220 & 4.220 & 4.220 & 4.220 & 4.220 & 4.220 \\
\hline$-\Delta \mathrm{G}\left(\mathrm{KJ} . \mathrm{mol}^{-1}\right)$ & 22.390 & 23.174 & 23.442 & 23.739 & 24.027 & 24.359 \\
\hline$\Delta \mathrm{S}\left(\mathrm{J} \cdot \mathrm{mol}^{-1} \cdot \mathrm{K}^{-1}\right)$ & 64.166 & 64.654 & 64.466 & 64.383 & 64.275 & 64.308 \\
\hline \multicolumn{7}{|c|}{$10 \%$ ethanol + $90 \%$ water } \\
\hline$-\Delta \mathrm{H}\left(\mathrm{KJ} . \mathrm{mol}^{-1}\right)$ & 22.124 & 22.124 & 22.124 & 22.124 & 22.124 & 22.124 \\
\hline$-\Delta \mathrm{G}\left(\mathrm{KJ} \cdot \mathrm{mol}^{-1}\right)$ & 24.471 & 23.880 & 24.203 & 24.282 & 24.660 & 24.434 \\
\hline$\Delta \mathrm{S}\left(\mathrm{J} \cdot \mathrm{mol}^{-1} \cdot \mathrm{K}^{-1}\right)$ & 8.289 & 5.992 & 6.974 & 7.118 & 8.230 & 7.377 \\
\hline \multicolumn{7}{|c|}{$15 \%$ ethanol $+85 \%$ water } \\
\hline$-\Delta \mathrm{H}$ & 14.625 & 14.625 & 14.625 & 14.625 & 14.625 & 14.625 \\
\hline$-\Delta \mathrm{G}(\mathrm{r}$ & 24.296 & 25.109 & 25.105 & 25.197 & 25.542 & 25.284 \\
\hline$\Delta \mathrm{S}(\mathrm{J}$. & 34.153 & 35.763 & 35.149 & 34.871 & 35.425 & 34.035 \\
\hline \multicolumn{7}{|c|}{$20 \%$ ethanol $+80 \%$ water } \\
\hline$-\Delta \mathrm{H}(\mathrm{KJ} . \mathrm{m}$ & 14.954 & 14.954 & 14.954 & 14.954 & 14.954 & 14.954 \\
\hline$-\Delta \mathrm{G}\left(\mathrm{KJ} \cdot \mathrm{mol}^{-1}\right)$ & 25.419 & 25.962 & 26.186 & 26.361 & 26.429 & 26.528 \\
\hline$\Delta \mathrm{S}\left(\mathrm{J} \cdot \mathrm{mol}^{-1} \cdot \mathrm{K}^{-1}\right)$ & 36.955 & 37.547 & 37.669 & 37.624 & 37.234 & 36.957 \\
\hline \multicolumn{7}{|c|}{$25 \%$ ethanol $+75 \%$ water } \\
\hline$-\Delta \mathrm{H}\left(\mathrm{KJ} \cdot \mathrm{mol}^{-1}\right)$ & 22.180 & 22.180 & 22.180 & 22.180 & 22.180 & 22.180 \\
\hline$-\Delta \mathrm{G}\left(\mathrm{KJ} \cdot \mathrm{mol}^{-1}\right)$ & 26.397 & 26.644 & 26.328 & 26.635 & 26.650 & 26.958 \\
\hline$\Delta \mathrm{S}\left(\mathrm{J} \cdot \mathrm{mol}^{-1} \cdot \mathrm{K}^{-1}\right)$ & 14.890 & 15.225 & 13.912 & 14.693 & 14.506 & 15.258 \\
\hline
\end{tabular}




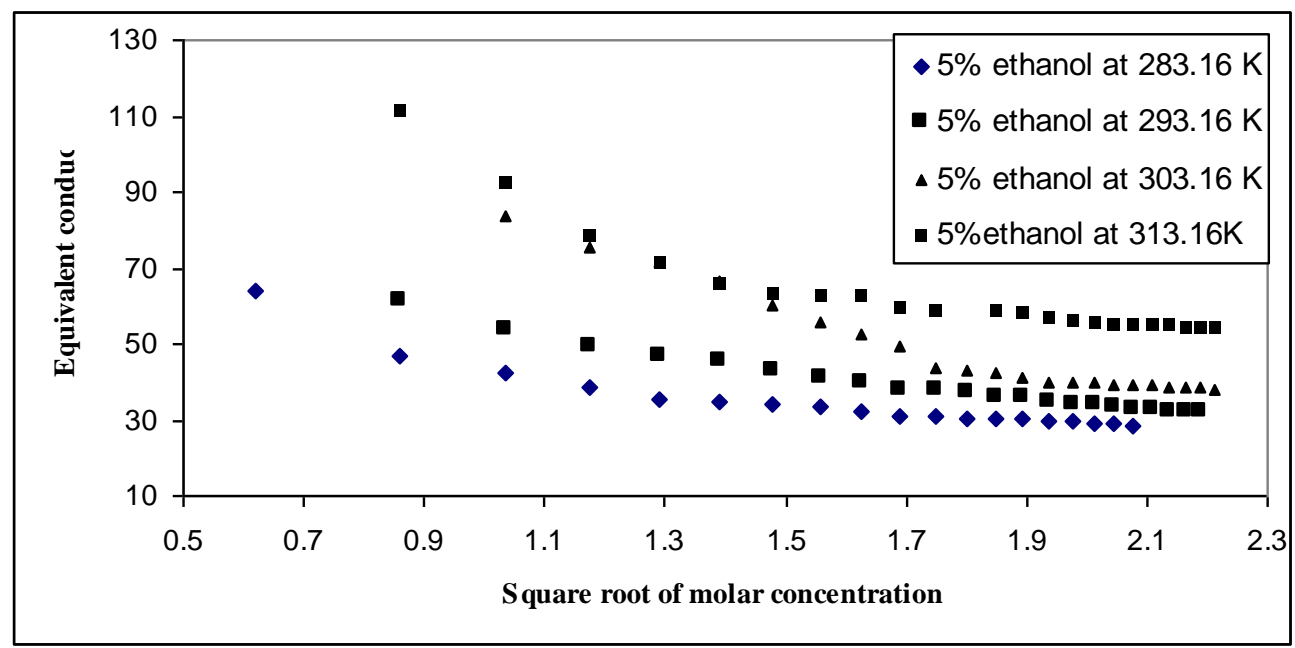

Figure 1: The plot of equivalent conductivity against the square root of molar concentration of acetyl acetonyl binyledene 5- amino salicylic acid in (5\% ethanol $+95 \%$ water) at different temperatures.

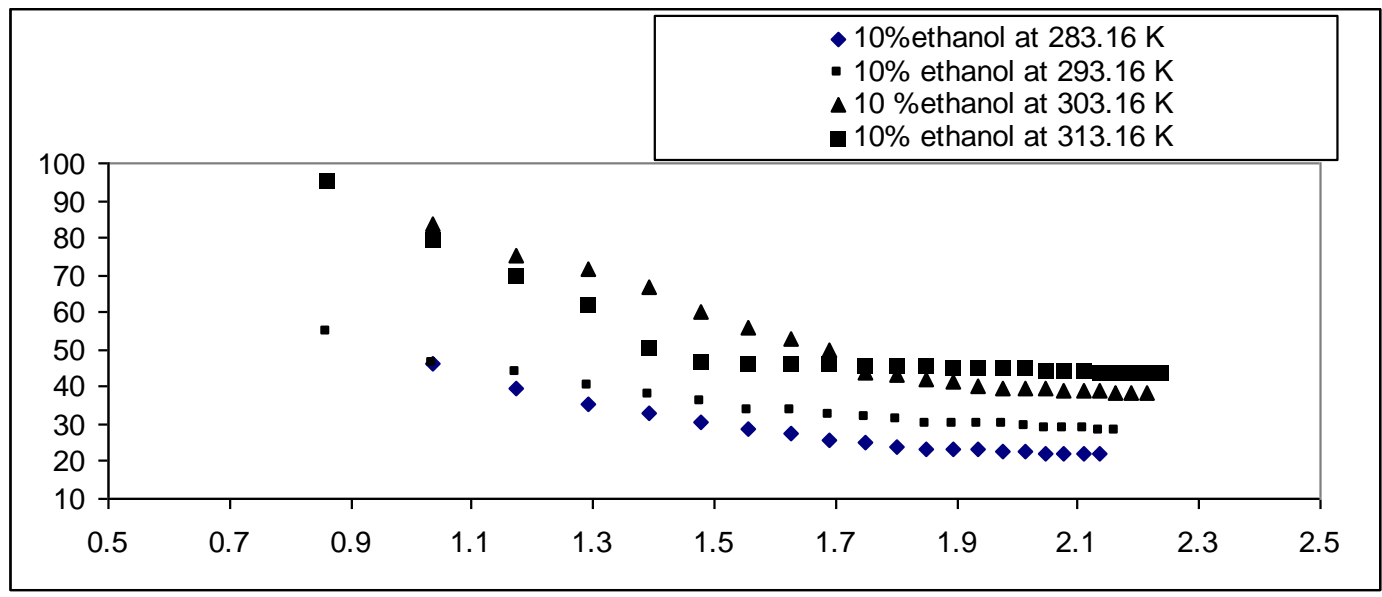

Figure 2: The plot of equivalent conductivity against the square root of molar concentration of acetyl acetonyl binyledene 5- amino salicylic acid in

(10 \% ethanol $+90 \%$ water) at different temperatures

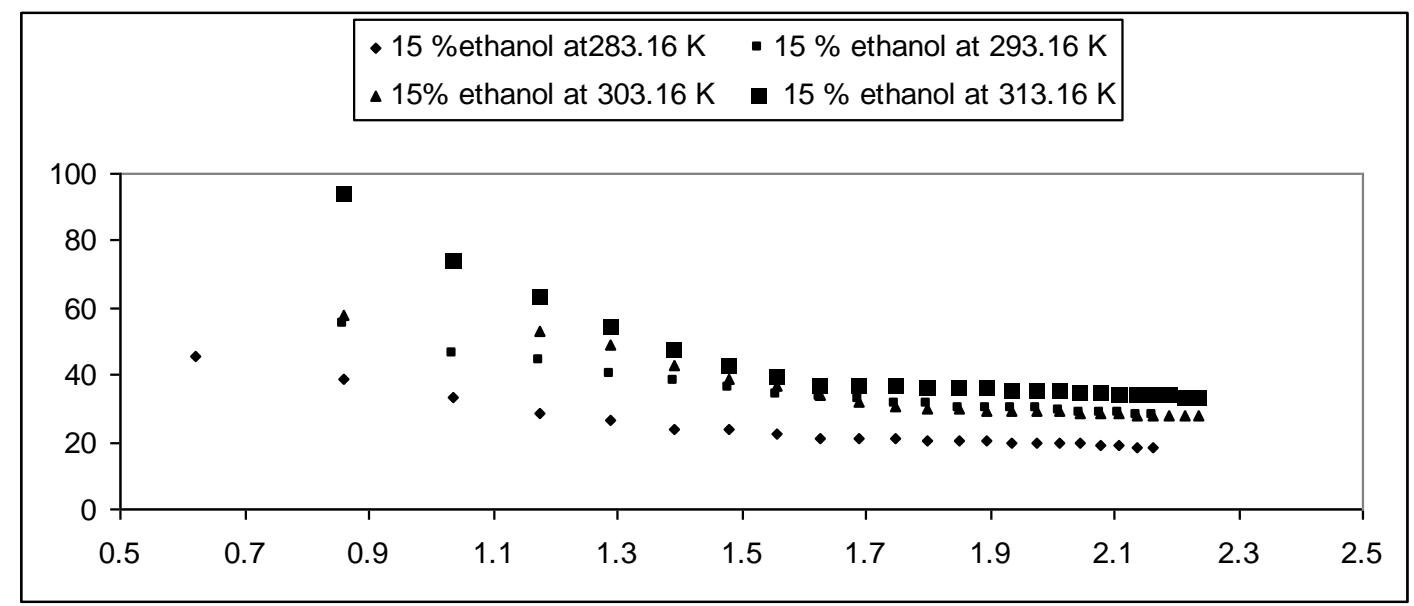

Figure 3: The plot of equivalent conductivity against the square root of molar concentration of acetyl acetonyl binyledene 5- amino salicylic acid in (15 $\%$ ethanol $+85 \%$ water) at different temperatures 


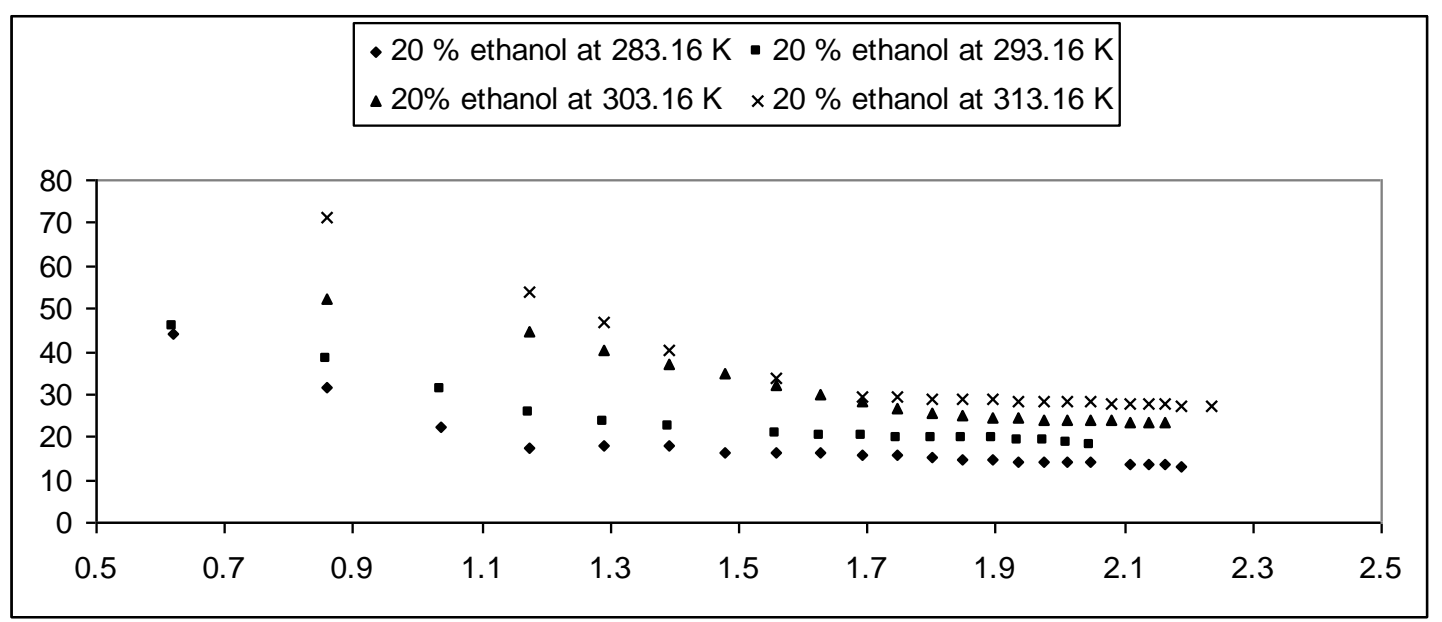

Figure 4: The plot of equivalent conductivity against the square root of molar concentration of acetyl acetonyl binyledene 5- amino salicylic acid in (20 \% ethanol $+\mathbf{8 0 \%}$ water) at different temperatures

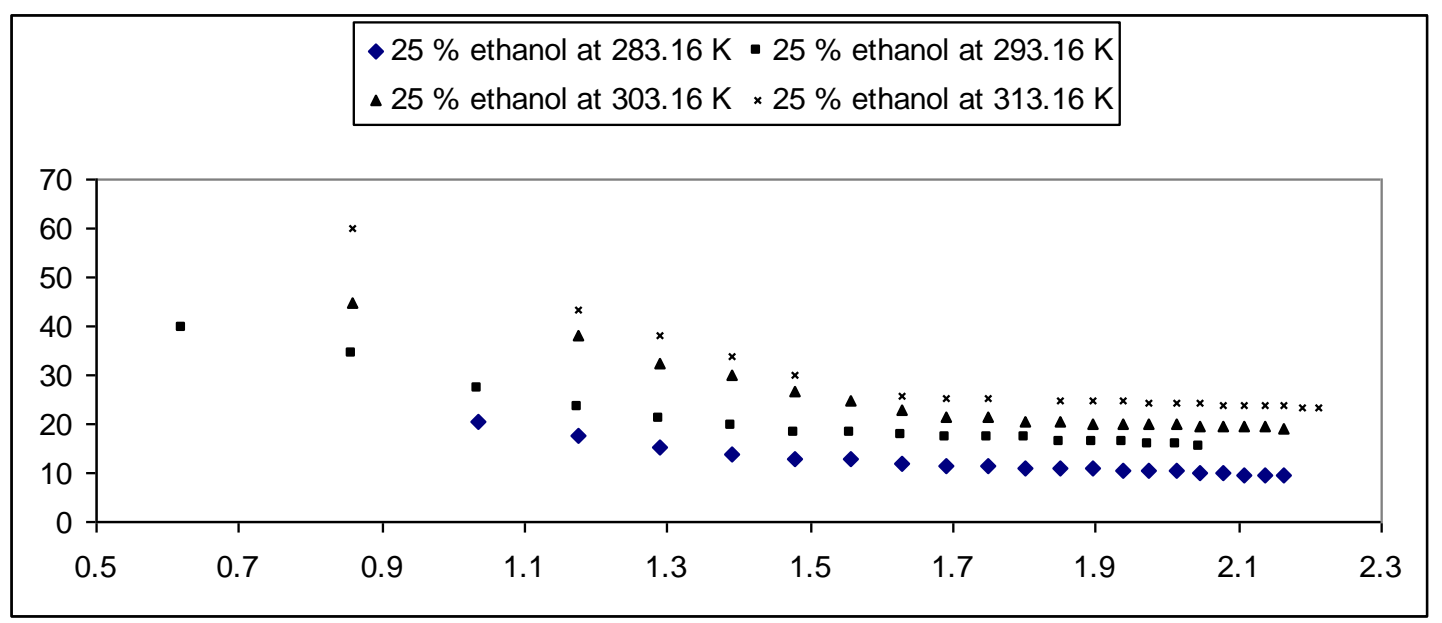

Figure 5: The plot of equivalent conductivity against the square root of molar concentration of acetyl acetonyl binyledene 5- amino salicylic acid in ( $25 \%$ ethanol $+75 \%$ water) at different temperatures

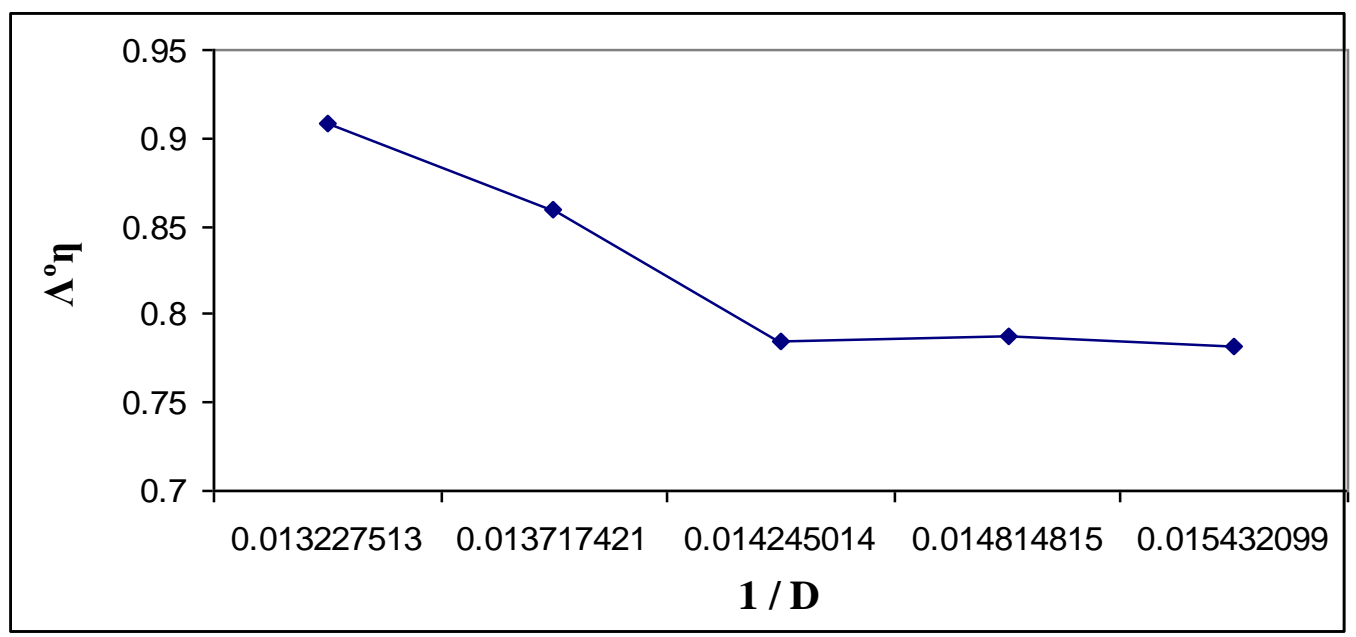

Figure 6: The plot of the Walden product against 1/D for acetyl acetonyl binyledene 5- amino salicylic acid in ethanol: water mixtures at $298^{\circ} \mathrm{K}$. 


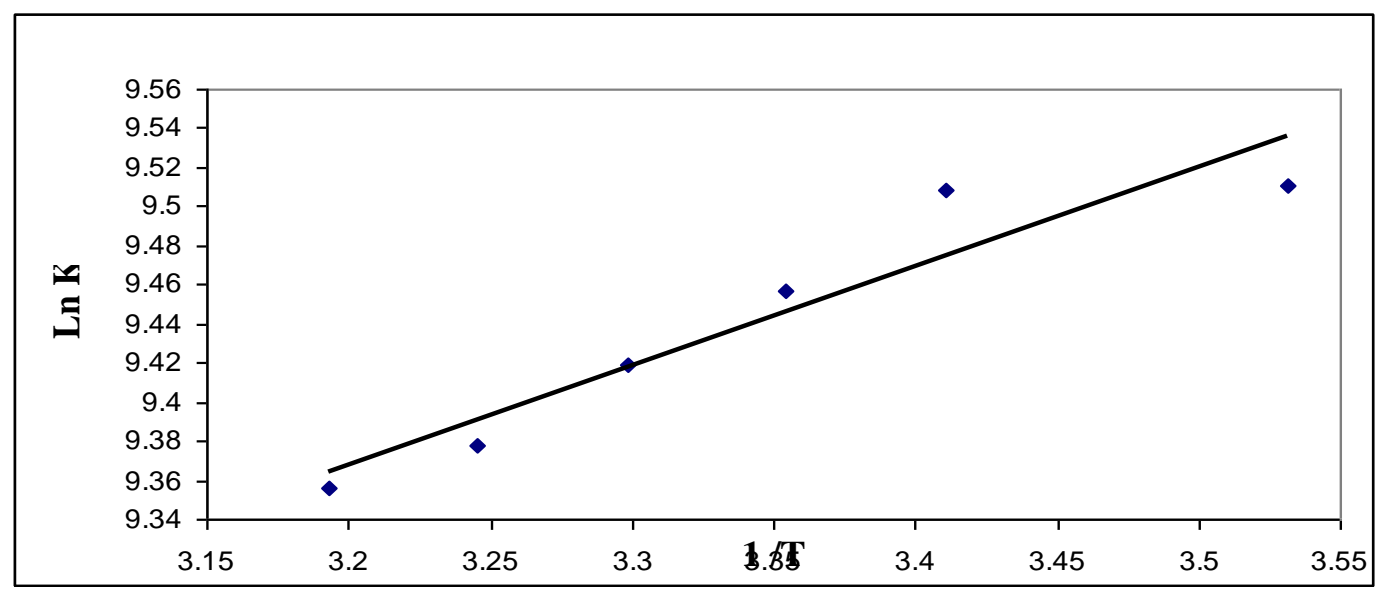

Figure 7: The plot of $\ln \left(K_{\mathrm{A}}\right)$ against $1 / \mathrm{T} * 10^{3}$ for acetyl acetonyl binyledene 5amino salicylic acid solution in $5 \%$ ethanol $+95 \%$ water mixture at different temperatures.

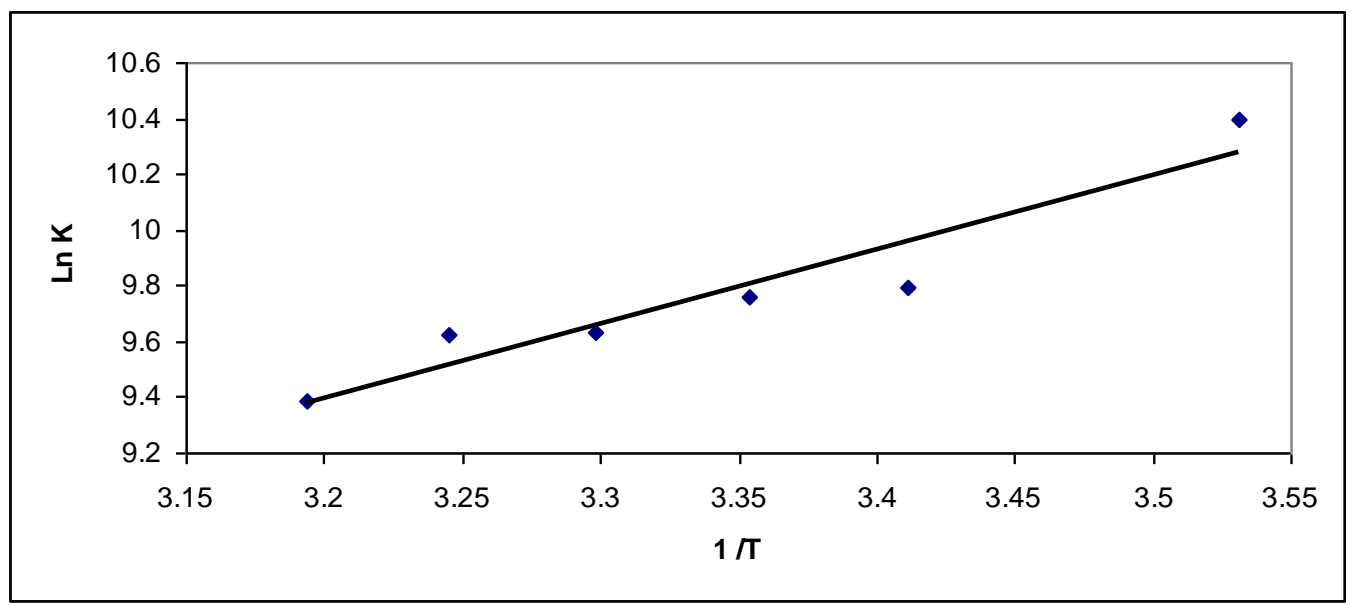

Figure 8: The plot of $\ln \left(K_{\mathrm{A}}\right)$ against $1 / \mathrm{T} * 10^{3}$ for acetyl acetonyl binyledene 5amino salicylic acid solution in $10 \%$ ethanol $+90 \%$ water mixture at different temperatures.

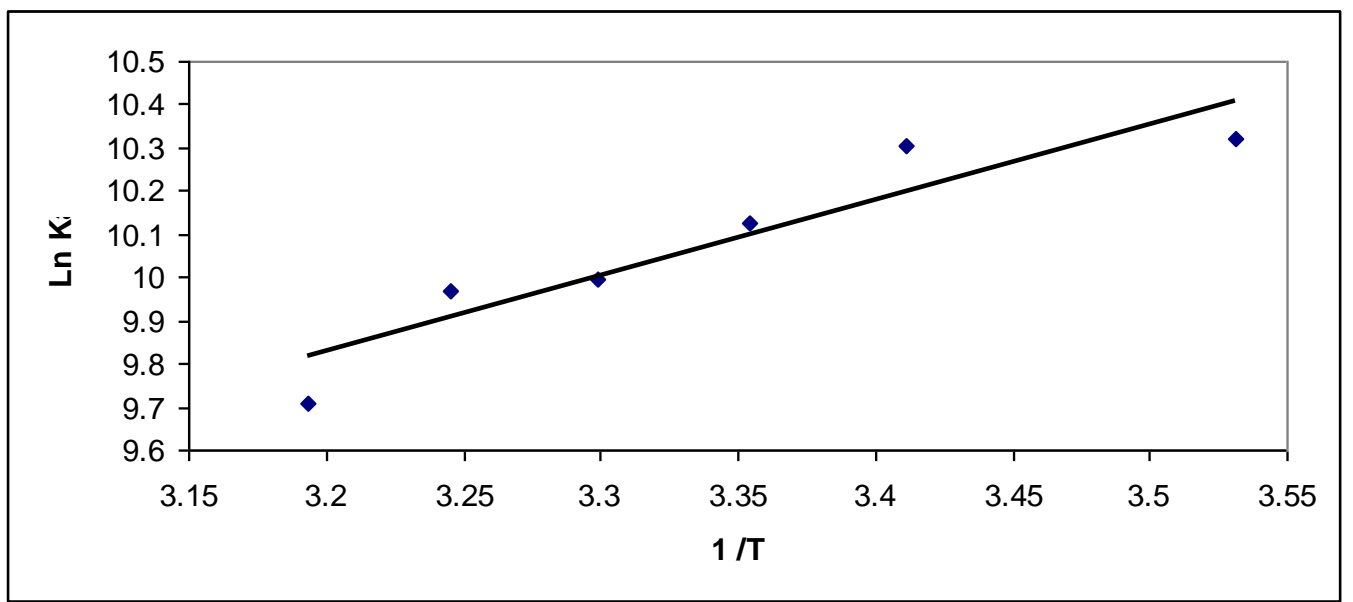

Figure 9: The plot of $\ln \left(K_{\mathrm{A}}\right)$ against $1 / \mathrm{T} * 10^{3}$ for acetyl acetonyl binyledene 5amino salicylic acid solution in $15 \%$ ethanol $+85 \%$ water mixture at different temperatures. 


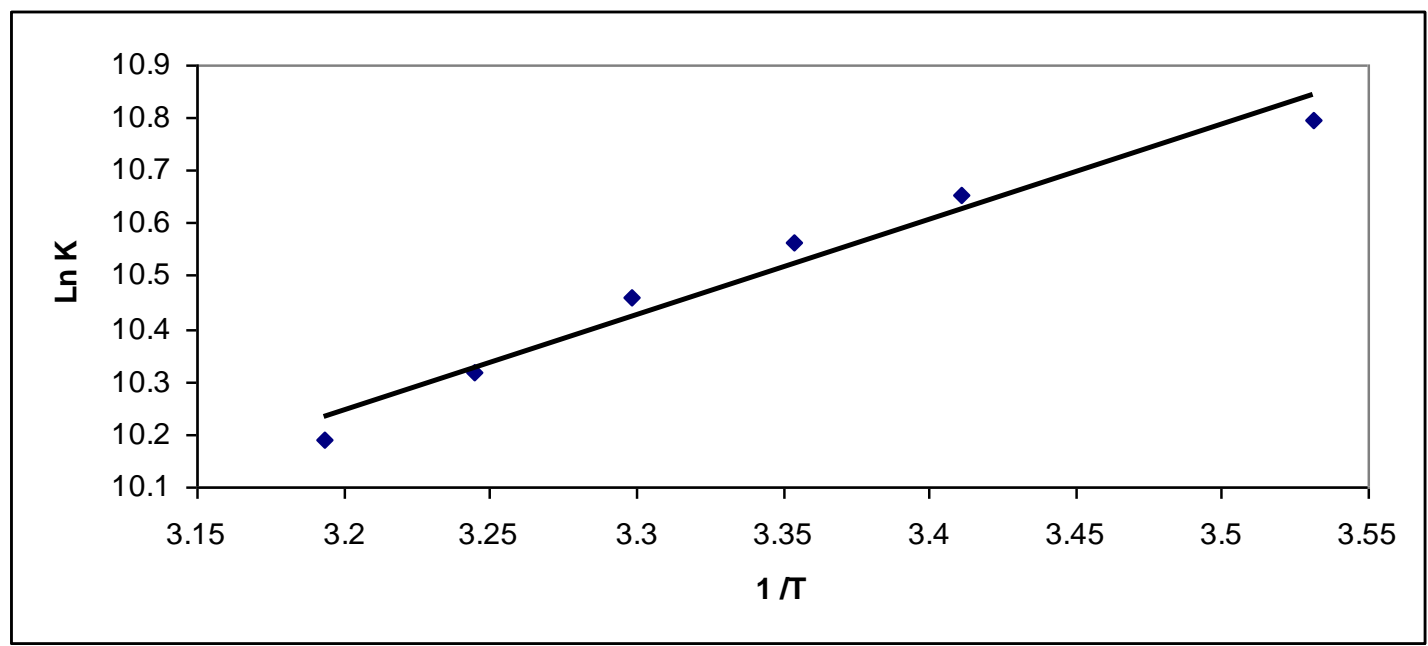

Figure 10: The plot of $\ln \left(K_{\mathrm{A}}\right)$ against $1 / \mathrm{T} * 10^{3}$ for acetyl acetonyl binyledene 5amino salicylic acid solution in $20 \%$ ethanol $+80 \%$ water mixture at different temperatures.

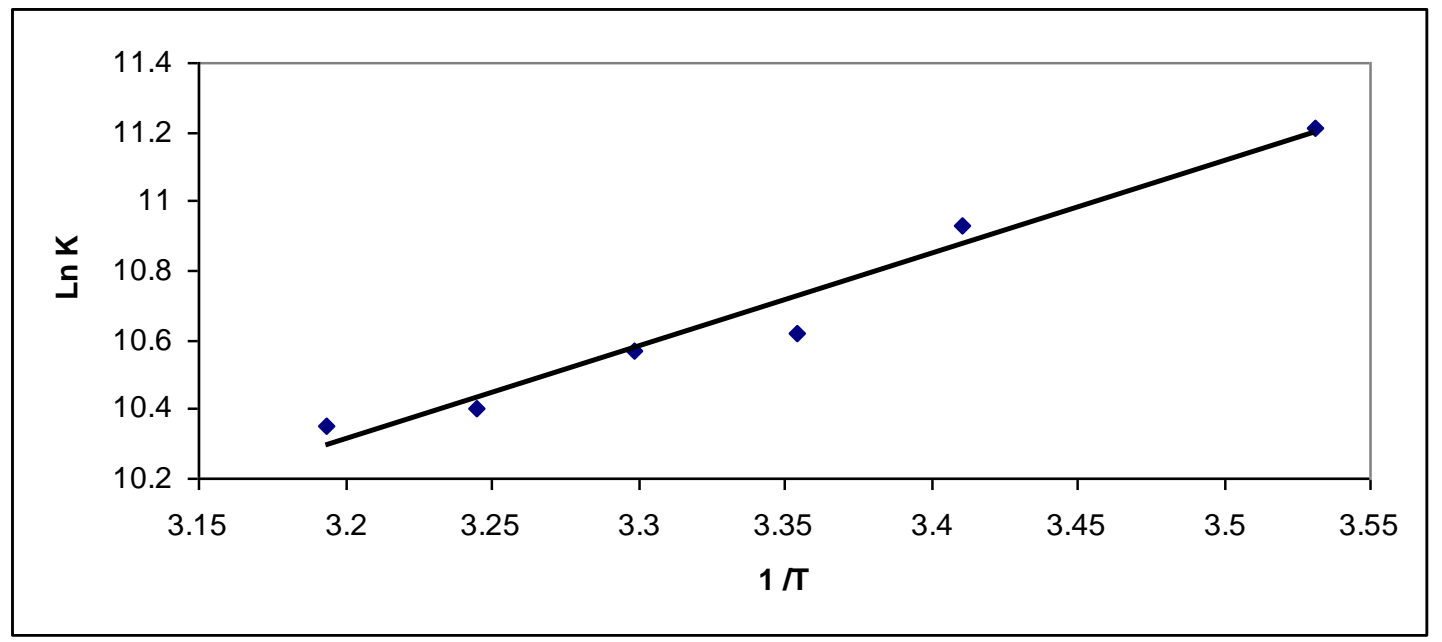

Figure 11: The plot of $\ln \left(K_{A}\right)$ against $1 / T * 10^{3}$ for acetyl acetonyl binyledene 5amino salicylic acid solution in $25 \%$ ethanol $+75 \%$ water mixture at different temperatures. 


\section{REFERENCES}

1) Fuoss. R. M., J.Phys. Chem., 80: 2091-2093 (1976).

2) Lee. W. H. and Wheaton. R. J., J. Chem. Soc. Faraday II., 74:743765 (1978).

3) Lee. W. H. and Wheaton. R. J., J. Chem. Soc. Faraday II., 74:14561482 (1978).

4) Lee. W. H. and Wheaton. R. J., J. Chem. Soc. Faraday II., 75:11281145 (1979).

5) Ansari. A. A and Islam. M. R., Can. J. Chem., 66:1223-1228 (1988).

6) Yokoyama. H., Ohta.T and Iida. M., Bull. Chem. Soc. Jpn., 65:2901-2909 (1992).

7) Tominic. I., Tomas. R., Visic M and Sokohi. V., Croatica. Chem. Acta., 77(3):537-543 (2004).

8) Tsierkeros. N. G and Molinon. I. E., J.S.C., 36(2):153-170 (2007).

9) El-Dossoki. F., J. Chem. Eng. Data., 55(6): 2155-2163 (2010).

10) A. I. Vogel, A Text Book of Practical Organic Chemistry, 1967, 3 rd ed., Longmans, London.

11) Akrawi. B. A., Ph.D Thesis, Surry University, Guildford (1981).

12) Pethybridge. A. D., and Taba. S. S., J. C. S., Faraday Trans., I 76: 368-376 (1980).

13) Hugo. V. H., Moran. J.A. and Sales. L. A., Can. J. Chem., 61:267268 (1983).

14) Glmeran. H. A., M.Sc. Thesis, College of science, University of Mosul (2007).

15) Akrawi. B. A., Abed. G. A., Q. SH. M., Raf. J. S., 17: 104-120 (2006).

16) Kon. H., Yokoyama. H., J. Phys. Chem., 95: 8956 (1991).

17) Hammes. P., J. Phys. Chem., 78., 907- 909 (1974).

18) Doe. H., Matoba. H., Ichimura and Kitagawa. T., Bull. Chem. Soc. Japan., 63: 2785-2789 (1990).

19) Al- Mastawi. Y. O. H., Ph.D. Thesis, College of science, University of Mosul (2002). 